\title{
Identification of host proteins interacting with the integrin-like A domain of Toxoplasma gondii micronemal protein MIC2 by yeast-two-hybrid screening
}

Yifan Wang ${ }^{1,2}$, Rui Fang ${ }^{1,2^{*}}$, Yuan Yuan ${ }^{1,2}$, Min Hu ${ }^{1,2}$, Yanqin Zhou ${ }^{2}$ and Junlong Zhao ${ }^{1,2^{*}}$

\begin{abstract}
Background: Toxoplasma gondii is an obligate intracellular protozoan, causing the important zoonosis toxoplasmosis. This parasite utilizes a unique form of locomotion called gliding motility to find and invade host cells. The micronemal adhesin MIC2 plays critical roles in these processes by binding to substrates and host cell receptors using its extracellular adhesive domains. Although MIC2 is known to mediate important interactions between parasites and host cells during invasion, the specific host proteins interacting with MIC2 have not been clearly identified. In this study, we used a yeast-two-hybrid system to search for host proteins that interact with MIC2.
\end{abstract}

Methods: Different adhesive domains of MIC2 were cloned into the PGBKT7 vector and expressed in fusion with the GAL4 DNA-binding domain as baits. Expression of bait proteins in yeast cells was analyzed by immuno-blotting and their autoactivation was tested via comparison with the pGBKT7 empty vector, which expressed the GAL4 DNA binding-domain only. To identify host proteins interacting with MIC2, a mouse cDNA library cloned into a GAL4 activation-domain expressing vector was screened by yeast-two-hybrid using the integrin-like A domain of MIC2 (residues 74-270) as bait. After initial screening and exclusion of false positive hits, positive preys were sequenced and analyzed using BLAST analysis and Gene Ontology Classifications.

Results: Two host proteins that had not previously been reported to interact with $T$. gondii MIC2 were identified: they are LAMTOR1 (late endosomal/lysosomal adaptor, MAPK and mTOR activator 1) and RNaseH2B (ribonuclease H2 subunit B). Gene Ontology analysis indicated that these two proteins are associated with many cellular processes, such as lysosome maturation, signaling transduction, and RNA catabolism.

Conclusion: This study is the first one to report interactions between Toxoplasma gondii MIC2 and two host proteins, LAMTOR1 and RNaseH2B. The data will help us to gain a better understanding of the function of MIC2 and suggest that MIC2 may play roles in modulating host signal transduction and other biological processes in addition to binding host cells.

Keywords: Toxoplasma gondii, MIC2, Integrin-like A domain, Yeast-two-hybird, LAMTOR1, RNaseH2B

\footnotetext{
*Correspondence: fangrui19810705@163.com; zhaojunlong@mail.hzau.edu.cn

'State Key Laboratory of Agricultural Microbiology, College of Veterinary

Medicine, Huazhong Agricultural University, Wuhan 430070, Hubei, PR China

${ }^{2}$ Key Laboratory of development of veterinary diagnostic products, Ministry

of Agriculture, Huazhong Agricultural University, Wuhan 430070, Hubei, PR

China
}

\section{Biomed Central}

(c) 2014 Wang et al.; licensee BioMed Central Ltd. This is an Open Access article distributed under the terms of the Creative Commons Attribution License (http://creativecommons.org/licenses/by/4.0), which permits unrestricted use, distribution, and reproduction in any medium, provided the original work is properly credited. The Creative Commons Public Domain Dedication waiver (http://creativecommons.org/publicdomain/zero/1.0/) applies to the data made available in this article, unless otherwise stated. 


\section{Background}

Toxoplasma gondii is an obligate intracellular protozan, which is responsible for toxoplasmosis in immunocompromised patient and livestock [1]. T. gondii is able to infect a wide variety of warm-blooded animals, including wildlife mammals [2], birds $[3,4]$ and humans. The broad host range of this parasite is partially due to its successful host invasion mechanism, which is conserved among many apicomplexan parasites [5]. Invasion is a multistep process that leads to the establishment of parasitophorous vacuoles (PV), in which the invaded parasites replicate within host cells [6]. T. gondii utilizes a unique mode of locomotion named "gliding motility" to get close to and actively penetrate host cells. Gliding motility is also responsible for tissue migration and local dissemination of the parasites. Powered by the actin-myosin motor complex, gliding motility also requires proteins released from the apical organelle called micronemes [7].

Previous studies have shown that micronemal proteins (MICs) of $T$. gondii have important roles in host-cell invasion [8]. The majority of MICs are adhesins, which bind to host cells during invasion. As one of the most extensively studied MICs," MIC2 is a transmembrane adhesin that plays crucial roles during gliding motility and host-cell invasion [9-12]. Although MIC2 is not absolutely essential [13], mutants lacking MIC2 display severe growth defects and are impaired in host cell attachment, helical gliding, host-cell invasion, and virulence in mice [12]. Disruption of M2AP, a micronemal protein tightly associated with $\mathrm{MIC} 2$, causes partial retention of MIC2 in the secretory pathway (i.e. ER/Golgi), which leads to reduced host cell binding [14]. Similarly, disruption of TRAP (a MIC2 ortholog) in Plasmodium berghei results in defects in motility and host-cell invasion in sporozoites $[15,16]$. Once released to the parasite surface from the micronemes, MIC2 undergoes proteolytical maturation by a parasite protease called MPP2 (microneme-processing protease 2), which trims the $\mathrm{N}$-terminus of MIC2 and activates its adhesive domains for substrate and receptor binding to promote gliding motility and invasion [17]. Mature MIC2 contains two different adhesive domains, a single von Willebrand factor A (vWA) integrin-like A domain (A/I) and a TSR domain containing six thrombospondin type I repeats. The A/I domain of MIC2 is able to interact with heparin, a ubiquitous glycosaminoglycan found in extracellular matrix of host cells [9]. In addition, MIC2 also interacts with intercellular adhesion molecule 1 (ICAM-1) via its $\mathrm{A} / \mathrm{I}$ domain to facilitate the migration of $T$. gondii across polarized epithelial cells [10]. Unlike the A/I domain, the host proteins interacting with the TSR domain of MIC2 are still unclear, although thrombospondin-1 is involved in interactions with many ligands in animals and humans [18].
Many studies have shown that binding of MIC2 to host cells promotes $T$. gondii invasion, however the host receptors that mediate $\mathrm{MIC} 2$ binding have not been identified. Therefore, identification of more host proteins that interact with $\mathrm{MIC} 2$ may help us better to understand the invasion mechanism and find targets for drug discovery. In the present study, a high throughput yeasttwo-hybrid screen was performed to search for host proteins that interact with T. gondii MIC2. Two hits, LAMTOR1 and RNaseH2B, which have not been reported previously to interact with $\mathrm{MIC} 2$, were identified using the A/I domain of MIC2 as bait. These two proteins are associated with many cellular processes in host cells, such as protein binding, endosome/lysosome maturation, signal transduction, RNA catabolic processes and so on. Thus, these findings indicate that MIC2 is not only involved in binding to host cells but may also affect host signal transduction and other biological processes.

\section{Methods}

\section{Mice and parasites}

Female KunMing mice aged 4-6 weeks were purchased from Laboratory Animals Research Centre of Hubei province, P. R. China. All the mice were raised under standard conditions and allowed access to feeding and water ad libitum. All animal experiments were performed under the approval of Laboratory Animals Research Centre of Hubei province and the ethics committee of Huazhong Agricultural University (Permit number: 4200695757).

T. gondii RH strain was maintained by peritoneal passage in KunMing mice. To be used in experiment for total RNA extraction, tachyzoites were harvested from peritoneal fluid of infected mice $3 \sim 4$ days post infection, and were purified by filtration through CF-11 cellulose (Whatman Inc., USA).

\section{Bait plasmids construction}

Total RNA from purified $T$. gondii tachyzoites was extracted using TRIzol Reagent according to manufacturer's instructions (Invitrogen, USA). One microgram of total RNA was reverse-transcribed into cDNA using the ReverTra Ace reverse transcription kit according to manufacturer's instructions (TOYOBO, Japan). The bait plasmids were constructed by cloning different fragments of MIC2 (TGGT1_210780, http://toxodb.org/toxo/) into the pGBKT7 vector. These MIC2 fragments include (see Figure 1A for illustration): the 1,569 bp fragment encoding a 523-residue (from aa74 to aa596) peptide of MIC2 ectodomain (to give pGBKT7-MIC2); the $591 \mathrm{bp}$ fragment encoding the A/I domain (197 amino acid residues, from aa74 to aa270) (to give pGBKT7-A/I); and the 978 bp fragment encoding the TSR domain (326 amino acid residues, from aa271 to aa596) (to give pGBKT7TSR). These fragments were PCR amplified from cDNA 


\section{A}

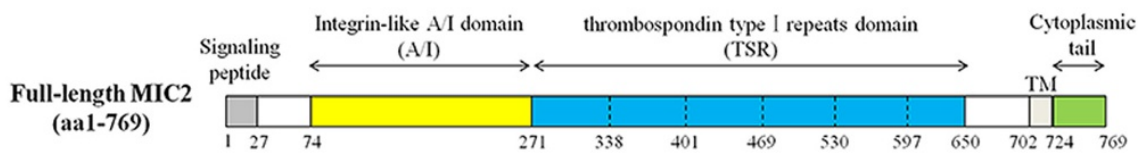

pGBKT7-MIC2

(aa74-596)

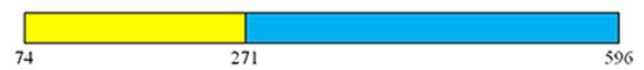

pGBKT7-A/I

(aa74-270)

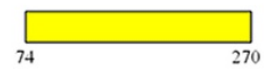

PGBKT7-TSR

(aa271-596)

B

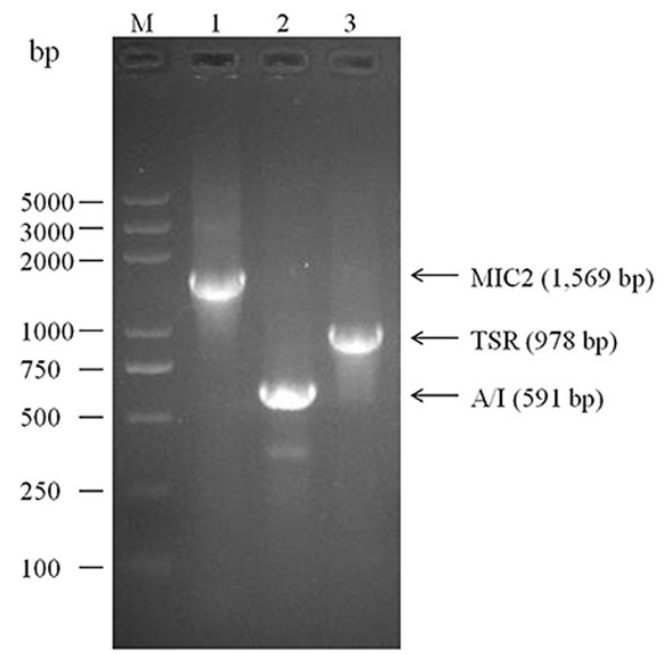

C

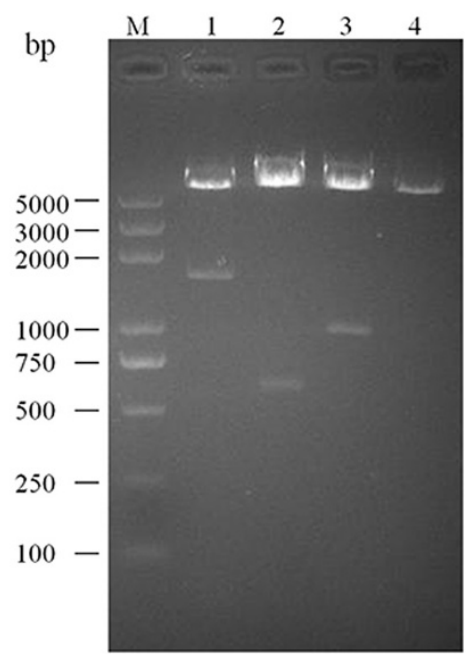

Figure 1 Construction of MIC2 bait plasmids. (A) Schematic illustration of full-length MIC2, and its different domains used as baits in the yeast-two-hybrid screen. (B) Agarose gel electrophoresis analysis of different fragments of MIC2 amplified from T. gondii cDNA. M: DNA maker; 1: MIC2 ectodomain (aa74 - 596) that lacks propeptide and the sixth TSR motif; 2: A/I domain of MIC2 (aa74 - 270); 3: the TSR domain containing five thrombospondin typelrepeats of MIC2 (aa271 - 596). (C) Agarose gel electrophoresis analysis of constructed bait plasmids digested with Ndel and Sall. M: DNA maker; 1: pGBKT7-MIC2; 2: pGBKT7-A/l; 3: pGBKT7-TSR; 4: pGBKT7 vector.

using corresponding primers listed in Table 1 and cloned into pGBKT7 between NdeI and SalI sites.

\section{MIC2 Baits expression in yeast cells}

To check the expression of MIC2 baits in yeast cells, each bait plasmid was transformed into Saccharomyces cerevisiae strain Y2HGold (MATa, trp1-901, leu2-3, 112, ura3-52, his3-200, gal4A, gal80A, LYS2 : : GAL1 ${ }_{U A S^{-}}$

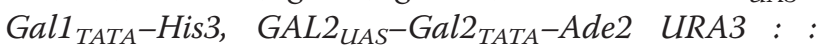
MEL1 $1_{\text {UAS }}-$ Mel1 $_{\text {TATA }}$ AUR1-C MEL1) using the lithium acetate method according to the User's Manual of Yeastmaker $^{\mathrm{TM}}$ Yeast Transformation System 2 (Cat. No. 630439, Clontech, USA). Transformants were selected on plates containing the minimal yeast medium without tryptophan (SD/-Trp) for $3 \sim 5$ days. Subsequently one colony from the SD/-Trp plate was inoculated into SD/Trp broth and grown until the $\mathrm{OD}_{600}$ reached 0.6. Then total proteins were extracted from pelleted cells by the Urea/SDS method [19]. Extracted proteins were then separated on 12\% SDS-PAGE gels and transferred electrophoretically to PVDF membrane (Millipore., USA) for Western blotting analysis. The MIC2 baits were c-Myc tagged and were detected with a mouse anti-Myc antibody (Medical and Biological Laboratories Co., Ltd., Japan) followed by peroxidase-conjugated goat anti-mouse secondary antibody (Beyotime., China). The blot was developed using the direct ECL chemiluminescent method (Thermo scientific, USA).

\section{Autoactivation test}

To test autoactivation of the bait proteins, each MIC2 bait plasmid was transformed into the yeast strain Y2HGold as above. Transformants were then grown on plates containing the minimal yeast medium without 
Table 1 Primers used to amplify different domains of MIC2 from CDNA of $T$. gondii tachyzoites

\begin{tabular}{lll}
\hline Primer name & Sequence $^{1}$ & $\begin{array}{l}\text { Restriction } \\
\text { enzyme sites }\end{array}$ \\
\hline pGBKT7-MIC2-F & GC CATATG CAGCTGGACATTGCTTC & Ndel \\
pGBKT7-MIC2-R & TA GTCGAC GACAAGCAGGATACGAACATG & Sall \\
pGBKT7-A/I-R & TA GTCGAC TGGCATCCTGGGGGAGTGTCTT & Sall \\
pGBKT7-TSR-F & GC CATATG GCCATTTGCTCGGATTGGTC & Ndel \\
\hline
\end{tabular}

${ }^{1}$ Restriction enzyme sites were presented in italics. Primers were used in the following combinations for PCR amplifications: pGKBT7-MIC2-F and pGKBT7-MIC2-R were used to amplify MIC2 ectodomain (aa74 - 596); pGBKT7-MIC2-F and pGKBT7-A/I-R were used to amplify the A/I domain (aa74 - 270); and pGKBT7-TSR-F and pGKBT7-MIC2-R were used to amplify the TSR domain (aa271 - 596).

tryptophan (SD/-Trp), or SD/-Trp supplemented with $40 \mathrm{ug} / \mathrm{ml} \mathrm{X- \alpha -Gal} \mathrm{(SD/-Trp/X),} \mathrm{or} \mathrm{SD/-Trp} \mathrm{supplemented}$ with $40 \mathrm{ug} / \mathrm{ml} \mathrm{X- \alpha -Gal} \mathrm{and} 125 \mathrm{ng} / \mathrm{ml}$ Aureobasidin A (SD/-Trp/X/A). Lack of autoactivation was indicated by white colonies on $\mathrm{SD} /$-Trp and $\mathrm{SD} /$-Trp/X plates and absence of colony growth on SD/-Trp/X/A plates. The bait that did not possess autoactivation activity was used in the yeast-two-hybrid screen.

\section{Yeast-two-hybrid screen}

All yeast strains, reagents and methods for yeast-twohybrid assays were from the Matchmaker ${ }^{\mathrm{Tx}}$ Gold Yeast Two-Hybrid System (Cat. No. 630489, Clontech, USA). To screen host proteins that interact with the A/I domain of MIC2, S.cerevisiae Y187 cells (MATa, ura352, his3-200, ade2-101, trp1-901, leu2-3, 112, gal4A, gal80A, met-, URA3 : : GAL1 $1_{U A S}-G a l 1_{T A T A}-L a c Z$, MEL1) containing the Mate \& Plate ${ }^{\mathrm{TM}}$ Universal Mouse (Normalized) cDNA library (Cat. No. 630482, Clontech., USA) cloned into the pGADT7-RecAB vector were used to mate with the Y2HGold cells transformed with the bait plasmid for $24 \mathrm{~h}$ at $30^{\circ} \mathrm{C}$. The mated culture was then spread onto $\mathrm{SD} /$-Leu/-Trp plates supplemented with $40 \mathrm{ug} / \mathrm{ml} \mathrm{X- \alpha -Gal} \mathrm{and} 125 \mathrm{ng} / \mathrm{ml}$ Aureobasidin A (DDO/ $\mathrm{X} / \mathrm{A}$ ). Blue colonies (which were potential positive hits) were restreaked onto higher stringency $\mathrm{SD} /$-Ade/-His/Leu/-Trp plates supplemented with $40 \mathrm{ug} / \mathrm{ml} \mathrm{X- \alpha -Gal} \mathrm{and}$ $125 \mathrm{ng} / \mathrm{ml}$ Aureobasidin A (QDO/X/A). The prey plasmids in positive clones were isolated using TIANprep yeast plasmid DNA kit (TIANGEN, China) and purified by transformation of $E$. coli DH5 $\alpha$ cells followed by selection on LB/Amp plates. To estimate the sizes of the specific inserts on positive prey plasmids, PCR analysis was carried out using the primers provided by Matchmaker ${ }^{\text {Tx }}$ AD LD-Insert Screening Amplimer Set (Cat. No. 630433, Clontech, USA). To exclude false positive hits, each putatively positive prey plasmid from the initial screen was cotransformed into Y2HGold strain with the bait plasmid. Transformants were then spread onto QDO/X/A plates to test for interactions. True positive hits were indicated by blue colonies under these conditions.

\section{Positive prey analysis}

The positive prey plasmids were further characterized by determining the DNA sequences of the inserts using Sanger sequencing. The sequencing results of positive hits were used in BLAST search to identify the corresponding mouse genes. Gene Ontology Classifications of identified genes were analyzed using the Mouse Genome Informatics database (http://www.informatics.jax.org/).

\section{Results \\ Construction of bait plasmids expressing different domains of MIC2}

The coding sequence of MIC2 ectodomain, which lacks propeptide and the sixth (last) TSR motif, was amplified from tachyzoites cDNA (Figure 1B lane 1), and cloned into pGBKT7 between the NdeI and SalI sites to be expressed in frame with the GAL4 DNA-binding domain. We also constructed two additional baits which express either the A/I domain or the TSR domain of MIC2, respectively. The coding sequences of the two domains were amplified from tachyzoites cDNA (Figure 1B lane 2 and 3) and cloned into pGBKT7 as above. Successful construction of these plasmids was confirmed by restriction enzyme digestion analysis (Figure 1C).

\section{Expression and auto-activation test of MIC2 baits in yeast cells}

Prior to yeast-two-hybrid screening, expression of the baits in yeast Saccharomyces cerevisiae was examined. Total proteins of the yeast strain Y2HGold pre-transformed with each corresponding bait plasmid (pGBKT7-MIC2, or pGBKT7-A/I, or pGBKT7-TSR) were extracted and analyzed by Western blotting using the Myc-tag antibody. The results indicated that all three bait proteins were expressed and easily detected by immuno-blotting. The MIC2 ectodomain bait fusion was detected to have a molecular weight $(\mathrm{MW})$ around $78 \mathrm{kDa}$, which is consistent with its calculated size (Figure 2A lane 1). Similarly, the A/I domain and the TSR domain fusions were detected around $43 \mathrm{kDa}$ and $56 \mathrm{kDa}$, respectively (Figure 2A lane 2 and 3). As a control, the pGBKT7 empty vector transformed Y2HGold cells expressed the GAL4 DNA-binding domain that was $21 \mathrm{kDa}$ in size (Figure 2A lane 4).

To test the autoactivation activity of these bait proteins in yeast cells, each bait plasmid was individually transformed into Y2HGold cells and subsequently the transformants were grown on minimal yeast media plates (i.e. SD/-Trp, SD/-Trp/X and SD/-Trp/X/A) to test autoactivation (Figure 2B). Autoactivation activities from the baits would enable the expression of reporter genes and lead to blue colonies on $\mathrm{SD} /-\mathrm{Trp} / \mathrm{X}$ plates and $\mathrm{SD} /-\mathrm{Trp} / \mathrm{X} / \mathrm{A}$ 
A

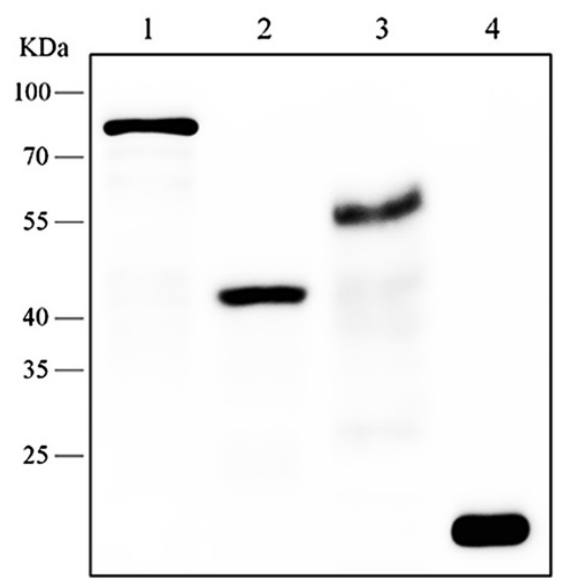

B

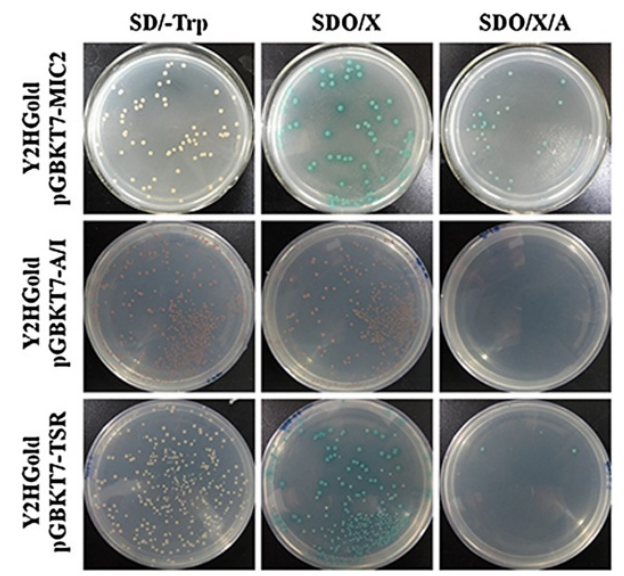

Figure 2 Expression and autoactivation of MIC2 baits in yeast cells. (A) Western blotting analysis on lysate of the yeast strain Y2HGold containing the following MIC2 bait plasmids. 1: pGBKT7-MIC2; 2: pGBKT7-A/I; 3: pGBKT7-TSR; 4: pGBKT7. (B) Determination of the auto-activation activity of different MIC2 baits in yeast cells.

plates. By doing so, we detected robust autoactivation activity for MIC2 ectodomain (A/I domain + TSR domain) and the TSR domain of MIC2 (Figure 2B). No autoactivation activity was detected from the A/I domain (Figure 2B), suggesting that the TSR domain was responsible for the autoactivation activity of the MIC2 ectodomain. According to these results, only the bait containing the A/I domain of MIC2 was used in the yeast-two-hybrid screen described below.

\section{Yeast-two-hybrid screen against a mouse cDNA library using the $\mathrm{A} / \mathrm{I}$ domain of MIC2 as bait}

To search for host proteins that interact with the A/I domain of MIC2 by yeast-two-hybrid, Y2HGold cells harboring the pGBKT7-A/I plasmid was used to mate with Y187 cells containing the Mate \& Plate $^{\mathrm{TM}}$ Universal Mouse (Normalized) cDNA library. After mating and growth on different selection plates, the mating efficiency was calculated to be $9.68 \%$ (data not shown). Based on this efficiency, we screened about $10^{9}$ viable diploid yeast cells. Among the $>200$ large colonies ( $2 \mathrm{~mm}-3 \mathrm{~mm}$ ) grown on $\mathrm{DDO} / \mathrm{X} / \mathrm{A}$ plates, twenty of them were blue, a phonotype that positive hits should have. Subsequently, these twenty blue clones were restreaked onto higher stringency QDO/X/A selection plates. Eight out of the twenty still gave blue colonies, indicating that they were likely to be positive hits. The prey plasmids were then isolated from these putatively positive clones and rescued through transformation of E.coli DH $5 \alpha$ cells. The specific insert on each prey plasmid was PCR amplified using primers originated from the vector backbones and analyzed by gel electrophoresis (Figure 3A). To eliminate false positive hits and retest the specificity of interaction, each of the eight prey plasmids was co-transformed with pGBKT7-A/I into Y2HGold cells and the co-transformants were tested on the QDO/X/A plates. The results indicated that two of the eight preys still showed positive interaction with the A/I bait but the other six did not (Figure 3B). To further check the interaction specificity of the two hits, we co-transformed each of the two prey plasmids with pGKBT7 empty vector into Y2HGold yeast cells and tested the growth and color of transformants on QDO/X/A plates. In both cases, we did not observe any colony growth on QDO/X/A plates, suggesting that both hits had specific interactions with the A/I domain of MIC2 (data not shown). Thus, two host proteins were identified to interact with the A/I domain of MIC2 and were further analyzed as below.

\section{Sequencing and analysis of positive prey}

To investigate the identity of host proteins in our positive hits, we sequenced the two prey plasmids using the T7 primer, which was located upstream of the inserts on the prey plasmids. The sequencing results (Additional file 1) were then used to search the mouse genome using the BLAST program (http://blast.ncbi.nlm.nih. gov/Blast.cgi). The results showed that the two inserts had high similarity $(99 \% \sim 100 \%)$ to two known genes of Mus musculus: the late endosomal/lysosomal adaptor MAPK and mTOR activator 1 (Lamtor 1, NM_025605) and the ribonuclease $\mathrm{H} 2$ subunit B (Rnaseh2b, NM_ 026001). Sequencing also showed that the Lamtor 1 containing hit had the full length Lamtor 1 on the plasmid in frame with the GAL4 DNA-binding domain, whereas the Rnaseh $2 b$ containing hit only had the Cterminus of the protein (from residue 111 to residue 308) (Additional file 1). These two proteins had not been reported to interact with $\mathrm{MIC} 2$ before. Gene ontology 


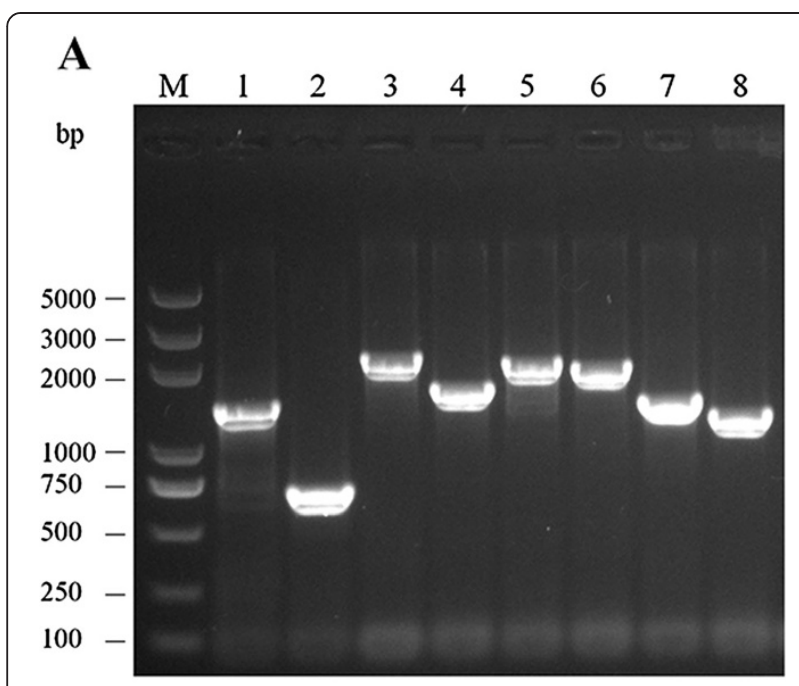

B

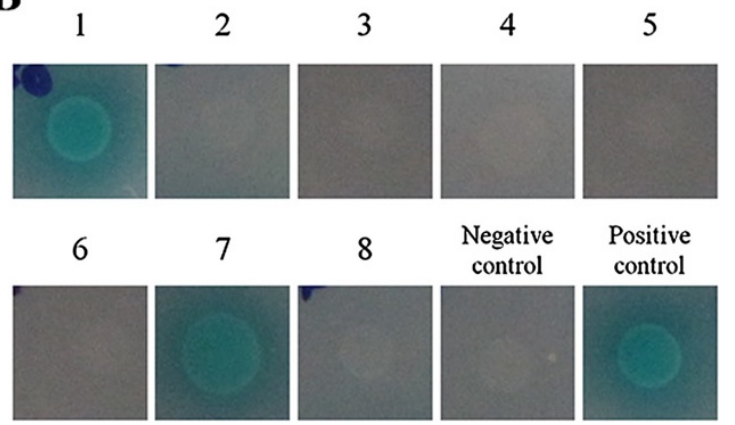

Figure 3 Analysis of putatively positive hits obtained from the yeast-two hybrid screen. (A) Agarose gel electrophoresis analysis of PCR products obtained from amplification of the inserts on putatively positive prey plasmids. M: DNA maker; Lane 1-8: PCR amplification products of the inserts on the eight putatively positive hits. (B) Confirmation of putative hits. Y2HGold cells were co-transformed with pGBKT7-AVI and each of the eight putatively positive prey plasmids (number 1 to 8 ) and plated on QDO/X/A plates; positive interaction was indicated by the presence of blue colonies. Co-transformation with pGADT7-T and pGBKT7-Lam was used as a negative control, whereas co-transformation with PGADT7-T and pGBKT7-53 was used as a positive control.

classifications and putative functions of these two proteins are shown in Table 2. The results indicated that LAMTOR1 functions as an adaptor involved in host signal transducation and $\mathrm{RNaseH} 2 \mathrm{~B}$ possesses ribonuclease activity during many cellular processes. Taken together, we discovered the interactions between MIC2 and two mouse proteins (LAMTOR1 and RNaseH2B), which may help understanding the cellular functions of MIC2 in greater detail.

\section{Discussion}

Although previous studies have demonstrated the interaction between $\mathrm{MIC} 2$ and host receptors during invasion
$[9,10]$, the specific host receptors involved in this interaction are still not clear. In order to identify host proteins that interact with MIC2, we took a high throughput yeast-two-hybrid screen strategy to search for mouse proteins that interact with MIC2. Three bait plasmids that expressed different parts of the MIC2 ectodomain were initially designed for the yeast-two-hybrid screen. Recent structural studies on Toxoplasma gondii MIC2 suggested that the MIC2 A/I domain can exist in both open and closed conformations [20]. Conversion of the closed conformation to the open conformation may occur after the cleavage of propeptide. Previous studies showed that open integrin I domains have higher receptor binding affinities than closed ones [21,22]. Thus, we thought that the MIC2 ectodomain lacking the propeptide would be a good bait to screen for binding partners, since it would be in an open conformation. In addition, the sixth TSR motif of the MIC2 ectodomain is only partially conserved and was found to be involved in M2AP binding [20]. Therefore, the sixth TSR motif was also excluded from our baits. In total, we constructed three baits: the A/I domain +5 TSR domains, the A/I domain alone and the 5 TSR domains alone.

Prior to the yeast-two hybrid screen, we tested the auto-activation activity of the three baits and found that the baits containing the 5 TSR domains showed autoactivation in yeast. This implies that the TSR domains may have eukaryotic transcriptional activation activities, which contributed to the auto-activation of MIC2 baits in yeast cells. To date, there are no data reporting interactions between the TSR domains of MIC2 and host proteins or carbohydrates, although its adhesive properties and motif conservation have been found in other species of apicomplexan parasites [23]. Thus, the TSR domains may play other roles for MIC2 function. Unlike the TSR domains, the A/I domain does not possess any detectable autoactivation activity and is expressed well in yeast cells. Therefore, we used the A/I domain of MIC2 as bait for our yeast-two hybrid screen.

Two novel mouse proteins, LAMTOR1 and RNaseH2B, were identified in our screen. This is the first study reporting interactions between MIC2 and these two host proteins. LAMTOR1 (late endosomal/lysosomal adaptor MAPK and mTOR activator 1), also called p18, is a membrane adaptor protein that localizes exclusively to the surface of lysosomes and late endosomes/lysosomes [24]. It serves as an anchor for the p14-MP1 complex that is involved in the late stages of lysosomal maturation, especially late endosome-lysosome fusion. LAMTOR1 and the LAMTOR1-p14-MP1 complex is highly conserved from yeast to humans [25]. Knockout of LAMTOR1 in mice leads to severe defects in endosome/lysosome organization and embryonic lethality during early developmental stages [24]. LAMTOR1 deficient cells show 
Table 2 The BLAST results and GO analysis of positive hits from the yeast-two-hybrid screen

\begin{tabular}{|c|c|c|c|}
\hline \multirow[t]{2}{*}{ Prey protein name } & \multirow{2}{*}{$\begin{array}{l}\text { Genbank } \\
\text { accession No. }\end{array}$} & \multicolumn{2}{|l|}{ Gene ontology classifications } \\
\hline & & Molecular function & Biological process \\
\hline \multirow[t]{8}{*}{$\begin{array}{l}\text { Late endosomal/lysosomal adaptor MAPK and } \\
\text { mTOR activatr } 1 \text { (LAMTOR1) }\end{array}$} & \multirow[t]{8}{*}{ NM_025605 } & $\begin{array}{l}\text { Guanyl-nucleotide exchange factor } \\
\text { activity }\end{array}$ & Cellular protein localization \\
\hline & & Protein binding & Cellular response to amino acid stimulus \\
\hline & & \multirow[t]{6}{*}{ Protein complex scaffold } & $\begin{array}{l}\text { Endosome/Lysosome localization and } \\
\text { organization }\end{array}$ \\
\hline & & & Positive regulation of MAPK cascade \\
\hline & & & Positive regulation of TOR signaling \\
\hline & & & Regulation of cholesterol homeostasis \\
\hline & & & Regulation of GTPase activity \\
\hline & & & Regulation of receptor recycling \\
\hline \multirow[t]{7}{*}{ Ribonuclease H2 subunit B (RNaseH2B) } & \multirow[t]{7}{*}{ NM_026001 } & \multirow{7}{*}{$\begin{array}{l}\text { RNA-DNA hybrid ribonuclease } \\
\text { activity }\end{array}$} & In utero embryonic development \\
\hline & & & Negative regulation of gene expression \\
\hline & & & Positive regulation of fibroblast proliferation \\
\hline & & & Regulation of DNA damage checkpoint \\
\hline & & & $\begin{array}{l}\text { Regulation of G2/M transition of mitotic } \\
\text { cell cycle }\end{array}$ \\
\hline & & & Ribonucleotide metabolic process \\
\hline & & & RNA catabolic process \\
\hline
\end{tabular}

aberrant distribution of lysosomes through perinuclear compartments, because of the exclusion of the p14-MP1 complex from the late endosomes. Recent studies show that the LAMTOR1-p14-MP1 complex is essential for regulating the activity of the mammalian Target Of Rapamycin Complex 1 (mTORC1) on lysosomes [26]. Conditional ablation of LAMTOR1 in mouse epidermis suggests that the LAMTOR1 related complex is involved in promoting lysosome-mediated degradation of cellular components, and is also required for regulating the formation of autolysosome [27]. Furthermore, LAMTOR1 also plays a potential role in $\mathrm{p} 53$-dependent apoptosis by regulating lysosomal activation [28]. Although still needs to be examined in vivo, our finding that MIC2 interacts with LAMTOR1 suggests that $T$. gondii infection may affect the above LAMTOR1-dependent host activities through MIC2- LAMTOR1 interaction.

The other mouse protein came out of our screen is RNaseH2B, a subunit of the ribonuclease (RNase) H2 enzyme complex, which shares high sequence homology in humans and mice [29]. The eukaryotic RNase H2 enzyme carries most of the RNase $\mathrm{H}$ activity in mammalian cells, which not only hydrolyzes the RNA strand of RNA/DNA hybrids but also recognizes single ribonucleotides in a DNA duplex and cleaves the 5'-phosphodiester bond of the ribonucleotide [29]. The RNase H2 complex consists of RNaseH2B and two other subunits: RNaseH2A and RNaseH2C. In this enzyme complex, the RNaseH2A subunit provides the main catalytic activity, whereas the $\mathrm{RNaseH} 2 \mathrm{~B}$ and $\mathrm{RNaseH} 2 \mathrm{C}$ subunits are possibly involved in interactions with other proteins [30-32]. RNaseH2B contains a PIP box at the C-terminus, which guides the interaction between RNase $\mathrm{H} 2$ enzyme complex and the proliferating cell nuclear antigen (PCNA), a protein involved in DNA replication [33]. Mutations in the genes encoding RNaseH2B subunits cause the AicardiGoutières syndrome (AGS), a neurological inflammatory disorder found in children [34]. This early-onset of neuroinflammation also has immunological similarities to the autoimmune disease systemic lupus erythematosus [35]. These observations suggest that RNaseH2B also plays crucial roles in the development of the nervous and immune systems. In addition, ablation of RNaseH2B in mice causes early embryonic lethality due to elevated DNA damage and reduced cell proliferation during gastrulation [36]. Given the multiple roles of RNaseH2B in host cells, Toxoplasma infection may modulate these $\mathrm{RNaseH} 2 \mathrm{~B}$ dependent processes through MIC2-RNaseH2B interaction, which deserves further investigation.

Although we have found two host proteins to interact with MIC2 from the yeast-two-hybrid screen, there are probably other host proteins that also interact with MIC2 but were not found in our screen, due to the limitations of this technology. For example, in this screen we did not find ICAM1, a protein known to interact with the A/I domain of MIC2. Possible explanations for this include: first, ICAM1 is a secretory protein associated with the membrane, which may be difficult to be localized 
to yeast nucleus, making it hard to be identified in the two-hybrid screen. Secondly, this Universal Mouse (Normalized) cDNA library may not contain any clones that express ICAM1 in the correct manner. Finally, previous studies have shown that multimerization of the $\mathrm{A} / \mathrm{I}$ domain is required for host cell binding [9]. However, this may not occur inside yeast cells. Given these limitations, it is possible that there are other MIC2 binding partners that were missed from our screen. Further efforts should be taken to identify these other interaction partners in the future to gain more insights into the function of MIC2.

\section{Conclusion}

Two host proteins, LAMTOR1 and RNaseH2B, which have not been previously reported to associate with Toxoplasma gondii infection, were identified by yeasttwo-hybrid screen to interact with MIC2 using the A/I domain of MIC2 as bait. These two host proteins are involved in many cellular processes, such as protein binding for signal transduction, lysosome maturation, RNA catabolism and DNA replication. Although the specific interactions between MIC2 and these two host proteins in vivo and the functional consequences of such interactions still need be confirmed further, the results from this study imply that micronemal proteins are not only involved in host cell binding but may also play roles in modulating host cell signaling and other biological processes during Toxoplasma gondii infection.

\section{Additional file}

Additional file 1: Figure S1. Sequencing results of the inserted fragments on positive hits. Sanger sequencing was carried out using the T7 sequencing primer. The sequences of the inserts obtained from sequencing reactions and the position of insertion on the vector are shown. (A) LAMTOR1 containing hit; (B) RnasH2B containing hit. The amino acid sequences of the corresponding proteins were underlined by dotted lines.

\section{Competing interests}

The authors declare that they have no competing interests.

\section{Authors' contributions}

JZ and RF conceived and designed the study, and critically revised the manuscript. YW carried out all of the experiments and drafted the manuscript. YY helped carry out yeast-two-hybrid screening. $\mathrm{MH}$ and $\mathrm{YZ}$ contributed to the revision of the manuscript. All authors read and approved the final manuscript.

\section{Acknowledgements}

We gratefully acknowledge the generous help from members of the State Key Laboratory of Agricultural Microbiology and the revision of manuscript from Dr. Shen. This work was funded by grants from National Natural Science Foundation of China (31101808), Specialized Research Fund for the Doctoral Program of Higher Education (20110146130001), National Key Basic Research Program (973 program) of China (Grant No. 2015CB150300) and Fundamental Research Funds for the Central Universities (2013PY059).
Received: 28 September 2014 Accepted: 17 November 2014

Published online: 26 November 2014

\section{References}

1. Tenter AM, Heckeroth AR, Weiss LM: Toxoplasma gondii: from animals to humans. Int J Parasitol 2000, 30:1217-1258.

2. Ferroglio E, Bosio F, Trisciuoglio A, Zanet S: Toxoplasma gondii in sympatric wild herbivores and carnivores: epidemiology of infection in the Western Alps. Parasit Vectors 2014, 7:196.

3. Cong W, Meng QF, Song HQ, Zhou DH, Huang SY, Qian AD, Su C, Zhu XQ: Seroprevalence and genetic characterization of Toxoplasma gondii in three species of pet birds in China. Parasit Vectors 2014, 7:152.

4. Tian YM, Dai FY, Huang SY, Deng ZH, Duan G, Zhou DH, Yang JF, Weng YB, Zhu XQ, Zou FC: First report of Toxoplasma gondii seroprevalence in peafowls in Yunnan Province, Southwestern China. Parasit Vectors 2012, 5:205.

5. Sibley LD: Intracellular parasite invasion strategies. Science 2004, 304:248-253.

6. Santos JM, Soldati-Favre D: Invasion factors are coupled to key signalling events leading to the establishment of infection in apicomplexan parasites. Cell Microbiol 2011, 13:787-796.

7. Sibley LD: How apicomplexan parasites move in and out of cells. Curr Opin Biotechnol 2010, 21:592-598.

8. Carruthers VB, Tomley FM: Microneme proteins in apicomplexans. Subcell Biochem 2008, 47:33-45.

9. Harper JM, Hoff EF, Carruthers VB: Multimerization of the Toxoplasma gondii MIC2 integrin-like A-domain is required for binding to heparin and human cells. Mol Biochem Parasitol 2004, 134:201-212.

10. Barragan A, Brossier F, Sibley LD: Transepithelial migration of Toxoplasma gondii involves an interaction of intercellular adhesion molecule 1 (ICAM-1) with the parasite adhesin MIC2. Cell Microbiol 2005, 7:561-568.

11. Brossier F, David Sibley L: Toxoplasma gondii: microneme protein MIC2. Int J Biochem Cell Biol 2005, 37:2266-2272.

12. Huynh MH, Carruthers VB: Toxoplasma MIC2 is a major determinant of invasion and virulence. PLOS Pathog 2006, 2:e84.

13. Andenmatten N, Egarter S, Jackson AJ, Jullien N, Herman JP, Meissner M: Conditional genome engineering in Toxoplasma gondii uncovers alternative invasion mechanisms. Nat Methods 2013, 10:125-127.

14. Huynh MH, Rabenau KE, Harper JM, Beatty WL, Sibley LD, Carruthers VB: Rapid invasion of host cells by Toxoplasma requires secretion of the MIC2-M2AP adhesive protein complex. EMBO I 2003, 22:2082-2090.

15. Sultan AA, Thathy V, Frevert U, Robson KJ, Crisanti A, Nussenzweig V, Nussenzweig RS, Menard R: TRAP is necessary for gliding motility and infectivity of plasmodium sporozoites. Cell 1997, 90:511-522.

16. Kappe S, Bruderer T, Gantt S, Fujioka H, Nussenzweig V, Menard R: Conservation of a gliding motility and cell invasion machinery in Apicomplexan parasites. J Cell Biol 1999, 147:937-944.

17. Carruthers VB, Sherman GD, Sibley LD: The Toxoplasma adhesive protein MIC2 is proteolytically processed at multiple sites by two parasitederived proteases. J Biol Chem 2000, 275:14346-14353.

18. Chen H, Herndon ME, Lawler J: The cell biology of thrombospondin-1. Matrix Biol 2000, 19:597-614.

19. Printen JA, Sprague GF Jr: Protein-protein interactions in the yeast pheromone response pathway: Ste5p interacts with all members of the MAP kinase cascade. Genetics 1994, 138:609-619.

20. Song G, Springer TA: Structures of the Toxoplasma gliding motility adhesin. Proc Natl Acad Sci U S A 2014, 111:4862-4867.

21. Shimaoka M, Xiao T, Liu JH, Yang Y, Dong Y, Jun CD, McCormack $A$, Zhang R, Joachimiak A, Takagi J, Wang JH, Springer TA: Structures of the alpha L I domain and its complex with ICAM-1 reveal a shapeshifting pathway for integrin regulation. Cell 2003, 112:99-111.

22. Schurpf T, Springer TA: Regulation of integrin affinity on cell surfaces. EMBO J 2011, 30:4712-4727.

23. Morahan BJ, Wang L, Coppel RL: No TRAP, no invasion. Trends Parasitol 2009, 25:77-84.

24. Nada S, Hondo A, Kasai A, Koike M, Saito K, Uchiyama Y, Okada M: The novel lipid raft adaptor p18 controls endosome dynamics by anchoring the MEK-ERK pathway to late endosomes. EMBO J 2009, 28:477-489.

25. Kogan K, Spear ED, Kaiser CA, Fass D: Structural conservation of components in the amino acid sensing branch of the TOR pathway in yeast and mammals. J Mol Biol 2010, 402:388-398. 
26. Takahashi Y, Nada S, Mori S, Soma-Nagae T, Oneyama C, Okada M: The late endosome/lysosome-anchored p18-mTORC1 pathway controls terminal maturation of lysosomes. Biochem Biophys Res Commun 2012, 417:1151-1157.

27. Soma-Nagae T, Nada S, Kitagawa M, Takahashi Y, Mori S, Oneyama C, Okada M: The lysosomal signaling anchor p18/LAMTOR1 controls epidermal development by regulating lysosome-mediated catabolic processes. J Cell Sci 2013, 126:3575-3584.

28. Malek M, Guillaumot P, Huber AL, Lebeau J, Petrilli V, Kfoury A, Mikaelian I, Renno T, Manie SN: LAMTOR1 depletion induces p53-dependent apoptosis via aberrant lysosomal activation. Cell Death Dis 2012, 3:e300.

29. Cerritelli SM, Crouch RJ: Ribonuclease H: the enzymes in eukaryotes. FEBS J 2009, 276:1494-1505.

30. Shaban NM, Harvey S, Perrino FW, Hollis T: The structure of the mammalian RNase $\mathrm{H} 2$ complex provides insight into RNA.DNA hybrid processing to prevent immune dysfunction. J Biol Chem 2010, 285:3617-3624.

31. Figiel M, Chon H, Cerritelli SM, Cybulska M, Crouch RJ, Nowotny M: The structural and biochemical characterization of human RNase $\mathrm{H} 2$ complex reveals the molecular basis for substrate recognition and AicardiGoutières syndrome defects. J Biol Chem 2011, 286:10540-10550.

32. Reijns MA, Bubeck D, Gibson LC, Graham SC, Baillie GS, Jones EY, Jackson AP: The structure of the human RNase $\mathrm{H} 2$ complex defines key interaction interfaces relevant to enzyme function and human disease. J Biol Chem 2011, 286:10530-10539.

33. Chon H, Vassilev A, DePamphilis ML, Zhao Y, Zhang J, Burgers PM, Crouch RJ, Cerritelli SM: Contributions of the two accessory subunits, RNASEH2B and RNASEH2C, to the activity and properties of the human RNase $\mathrm{H} 2$ complex. Nucleic Acids Res 2009, 37:96-110

34. Rice Gl, Forte GM, Szynkiewicz M, Chase DS, Aeby A, Abdel-Hamid MS, Ackroyd S, Allcock R, Bailey KM, Balottin U, Barnerias C, Bernard G, Bodemer C, Botella MP, Cereda C, Chandler KE, Dabydeen L, Dale RC, De Laet C, De Goede CG, Del Toro M, Effat L, Enamorado NN, Fazzi E, Gener B, Haldre M, Lin JP, Livingston JH, Lourenco CM, Marques W Jr: Assessment of interferon-related biomarkers in Aicardi-Goutières syndrome associated with mutations in TREX1, RNASEH2A, RNASEH2B, RNASEH2C, SAMHD1, and ADAR: a case-control study. Lancet Neurol 2013, 12:1159-1169.

35. Ramantani $G$, Kohlhase J, Hertzberg $C$, Innes AM, Engel K, Hunger $S$, Borozdin W, Mah JK, Ungerath K, Walkenhorst H, Richardt HH, Buckard J, Bevot A, Siegel C, von Stulpnagel C, Ikonomidou C, Thomas K, Proud V, Niemann F, Wieczorek D, Hausler M, Niggemann P, Baltaci V, Conrad K, Lebon P, Lee-Kirsch MA: Expanding the phenotypic spectrum of lupus erythematosus in Aicardi-Goutières syndrome. Arthritis Rheum 2010, 62:1469-1477.

36. Reijns MA, Rabe B, Rigby RE, Mill P, Astell KR, Lettice LA, Boyle S, Leitch A, Keighren M, Kilanowski F, Devenney PS, Sexton D, Grimes G, Holt IJ, Hill RE, Taylor MS, Lawson KA, Dorin JR, Jackson AP: Enzymatic removal of ribonucleotides from DNA is essential for mammalian genome integrity and development. Cell 2012, 149:1008-1022.

doi:10.1186/s13071-014-0543-1

Cite this article as: Wang et al: Identification of host proteins interacting with the integrin-like A domain of Toxoplasma gondii micronemal protein MIC2 by yeast-two-hybrid screening. Parasites \& Vectors 2014 7:543.

\section{Submit your next manuscript to BioMed Central and take full advantage of:}

- Convenient online submission

- Thorough peer review

- No space constraints or color figure charges

- Immediate publication on acceptance

- Inclusion in PubMed, CAS, Scopus and Google Scholar

- Research which is freely available for redistribution

Submit your manuscript at www.biomedcentral.com/submit
() Biomed Central 